\title{
Identidades masculinas entre los Waorani y prácticas económicas en los últimos 50 años
}

David Hidrobo

RESUMEN

El PRESENTE TEXTO HACE UN BREVE ANÁLISIS DE LA CONSTRUCCIÓN DE IDENTIDAdES DE LOS HOMBRES WAORANI EN EL CONTEXTO DE LAS PRÁCTICAS ECONÓMICAS DE PRODUCCIÓN CIRCULACIÓN Y CONSUMO DURANTE LOS ÚLTIMOS 50 años. SE SEÑalan Mecanismos de IDENTIFICACIÓN Y RECONOCIMIENTO de LO PROPIO Y LO AJENO, EN UN CONTEXTO

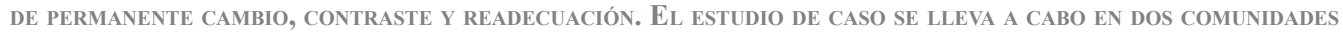
WAORANI QUE TIENEN UN PASADO COMÚN, PERO QUE HAN OPTADO POR FORMAS ECONÓMICAS DIFERENTES Y, EN ALGUNOS PUNTOS, OPUESTAS.

Palabras Clave: identidad - Reciprocidad - Petróleo - turismo - WaOrani.

Abstract

This TeXt is a BRIEF ANALysis of THE CONSTRUCTION OF IDENTITIES OF WAORANi MEN IN THE CONTEXT OF THE ECONOMIC PRACTICES OF PRODUCTION, CIRCULATION AND CONSUMPTION DURING THE LAST FIFTY YEARS. MECHANISMS OF IDENTIFICATION AND RECOGNITION OF OWNERSHIP AND NON- OWNERSHIP IN A CONTEXT OF PERMANENT CHANGE, CONTRAST AND REPURPosing. THE CASE DE STUdy WAS CARRIED OUT IN TWO WAORANi COMMUNITIES THAT SHARE A COMMON HISTORY BUT HAVE CHOSEN DIFFERENT AND SOMETIMES OPPOSITE ECONOMIC SYSTEMS.

KEY WORDS: IDENTITY - RECIPROCITY - OIL - TOURISM - WAORANI. 


\section{Introducción}

n los últimos 50 años, el grupo Waorani ubicado en la región nororiental del Ecuador ha experimentado profundos cambios que se expresan en las diferentes esferas de la vida de este pueblo. El siguiente artículo identifica y analiza las modificaciones y negociaciones que se han dado en la construcción de identidades de los hombres Waorani desde los escenarios relacionados a las prácticas económicas de producción, circulación y consumo de dos poblaciones waorani.

En el aspecto de la producción, se identifican prácticas económicas consideradas como tradicionales (cacería) o como nuevas (petróleo, turismo); con el objetivo de observar las implicaciones sociales que suscitan en el pueblo waorani en general y en las comunidades de estudio en particular.

Como elemento transversal de análisis, se analizan las dinámicas de reciprocidad entre actores waorani y no-waorani. En relación al tema de circulación, se identifica los grados de cercanía entre actores y la delimitación de fronteras entre los mismos, mediante el estudio de la reciprocidad generalizada, equilibrada y negativa, conceptos que fueron propuestos por Marshall Sahlins.

El estudio del consumo es abordado como consecuencia de la permanencia o cambio de las prácticas productivas e incorpora nuevos elementos a la construcción de identidades.

Para la recopilación de la información se utilizó fuentes bibliográficas sobre el grupo en mención, pero también se incorporó datos de una investigación de campo levantada en el año 2009, en las comunidades de Keweiriono y Gareno, con financiamiento de la National Science Foundation (NSF).

\section{El grupo Waorani: características generales y actividades económicas relevantes}

El pueblo waorani se ubica en el nororiente ecuatoriano, ocupando las provincias de Pastaza, Napo y Orellana. Rival describe la región como parecida al bosque lluvioso tropical, "con estribaciones importantes en su borde occidental y estaciones menos marcadas de las que normalmente imperan en la Cuenca del Amazonas" (Rival, 1996. 14). Dentro del territorio waorani, se encuentra el área protegida del "Parque Nacional Yasuní" que cuenta con una extraordinaria diversidad biológica. Este lugar protege un amplio rango de la comunidad de vegetales arbóreos, con alrededor de 2244 especies de árboles y arbustos, donde viven alrededor del 40\% de todas las especies de mamíferos de la cuenca amazónica (Ibíd.: 120), alrededor de 567 especies de aves, 105 de anfibios, 83 de reptiles, 382 de peces y más de 100 mil especies de insectos por hectárea (amazoniaporlavida.org).

En relación al territorio, para 1958, año en que se concretó el primer contacto "pacífico" con grupos no waorani, el pueblo de estudio dominaba un espacio que oscilaba entre $13.162 \mathrm{~km} 2$ y $20.000 \mathrm{~km} 2$; y su población alcanzaba las 500 personas, es decir había un promedio de 0,25 personas por km2 (Yost; 1979; 1981).

La forma de organización en este tiempo, tenía como base al nanicabo (en waoterero grupo o conjunto) una unidad social compuesta de muchas familias emparentadas entre sí (Calderón, 1998) y que se materializaba en una residencia conjunta donde habita un grupo doméstico (Rival, 1996). El grupo huaomoni, era el conjunto de nanicabos que se reunían en torno a una gran persona llamada "Ahuene", reconocida por su capacidad para velar por la paz y generar alianzas entre los grupos. Los grupos huaomoni, compartían y cuidaban un territorio, celebraban fiestas juntos y se casaban entre sí (Ibíd.; 134). Se mantenían alejados de otros grupos waomoni de parentesco lejano (huarani) y en franca hostilidad con grupos no waorani (kowore).

Cabodevilla (1999) explica que esta forma de vida se dio en respuesta a situaciones violentas que se desataron por la actividad cauchera en las últimas décadas del s.XIX y primeras del s.XX. 
El caucho provocó la entrada de varios peones encargados de peinar toda la selva en busca de los árboles de goma o de indígenas libres para convertirlos en mano de obra barata. Las contra ofensivas waorani a esta invasión generaron en el imaginario de otros grupos indígenas, comerciantes caucheros y sociedad nacional la idea de este grupo como salvajes o "aucas".

En este ambiente de tensión, fueron detectados, pero no plenamente reconocidos los grupos wao. El reconocimiento oficial del grupo waorani tiene su inicio en 1958 con la entrada de misioneras evangélicas pertenecientes al Instituto Lingüístico de Verano (ILV), el cual ingresa legalmente a territorio ecuatoriano en 1952 (Rivas, 2001; Cabodevilla, 1999) con el objetivo formal de hacer estudios lingüísticos alrededor de diversos grupos indígenas. El primer intento de contacto se denominó "Operación Auca" y terminó con la muerte de 5 misioneros norteamericanos. Elizabeth Elliot, esposa de uno de los muertos y Raquel Saint, hermana de Nate, el organizador de la expedición, también muerto; continuaron con el proceso de contacto. Raquel Saint se convertiría en la líder de este propósito.

Para ingresar al territorio waorani, Saint y Elliot aprendieron a hablar waoterero ${ }^{2}$ con Dayuma, una de las jóvenes waorani que había huido de su territorio por guerras internas a la hacienda Ila, propiedad del patrón cauchero Carlos Sevilla. Después de varios preparativos, entran finalmente en 1958 a la zona alta del Curaray, donde se encontraba un mermado grupo de familiares de Dayuma, reunidos en torno a Guikita, guerrero que había participado en el ataque a los 5 misioneros. Desde esta época se establece la primera misión evangélica a orillas del río Tiweno y toma su nombre (Rivas, 2001; Cabodevilla, 1999). Tiweno se conformó con un protectorado con una extensión de $1007 \mathrm{~km} 2$. Para 1971, 525 waorani vivían en este lugar y quedaban tan solo 100 fuera de sus límites.

En los 20 años de existencia del protectorado, hubo un crecimiento poblacional considerable, ya que se conciliaron nuevos matrimonios y cesaron las guerras internas. Sin embargo, en 1968 se dio una epidemia de poliomelitis que desanimó a algunas personas a mantenerse en la comunidad. A más de la epidemia, en parte debido a la desconfianza entre grupos y a los desacuerdos con la dirigencia de Raquel Saint y Dayuma, algunos grupos inconformes comenzaron a salir de Tiweno a partir de 1971 (Stoll, 1985; Cabodevilla, 1999).

Algunos waorani se establecieron entonces en otros lugares de la forma tradicional, pero se abrieron también nuevos senderos de comunicación con el mundo exterior. De esta forma, el contacto con las misioneras del ILV inauguró un tiempo de variados e intensos encuentros con diferentes actores: petroleros, científicos, madereros, colonos y turistas.

En el año de 1983, Oswaldo Hurtado, Presidente en ese entonces del Ecuador, entregó 66.570 hectáreas y, en 1990, Rodrigo Borja añadió a éstas 612.569 hectáreas más, lo que suma 679.130 hectáreas que son de propiedad de este grupo étnico (Mondragón y Smith, comp., 1997: 17).

Después de la salida del ILV, varios agentes externos establecieron relaciones con el grupo waorani, entre ellos los relacionados a la actividad petrolera. Desde 1964, la Texaco Gulf reinició con fuerza las actividades que la Shell había dejado inconclusas y desde 1971 las intensificó. En 1976, la Compagnie Générale de Géophysique (CGG) establecida en Pañacocha a $150 \mathrm{~km}$ del Coca, río abajo, realizaba también trabajos de prospección en territorio waorani (Narváez, 2008). A partir de este momento, más de 16 compañías han operado en el territorio.

Los primeros años de explotación petrolera en el territorio waorani se caracterizaron por una constante tensión entre operarios petroleros y waorani. Monseñor Alejandro Labaka (2003) expresa estos problemas:

"El día anterior [los trabajadores de la compañía] habían recibido la visita de "los amigos" (los llaman así porque al llegar al campamento saludaron diciendo "amigos"). Después se dedicaron a requisar todo el campamento, mientras uno de ellos repetía: "Pañacocha, Pañacocha, cambio...".

1 Palabra quichua que significa salvaje.

2 El idioma del pueblo waorani. 
Se llevaron cuanto se les antojó: hamacas, mantas, mosquiteros, ropa, botas, hachas, machetes, limas; de alimentos, todo lo que había de azúcar, un poco de arroz, latas de sardina y atún, y hasta dinero por valor de más de 3000 sucres. Uno de ellos, como contando el dinero con sus dedos dijo: "to,to,to...... Quito". (Ibíd.: 16-17)

Este tipo de situaciones decreció en la medida que diferentes personas waorani, en especial hombres, entraron en una relación laboral con las compañías petroleras y estas últimas, fueron armando planes y estrategias de comunicación social para este grupo. Aunque la presencia de los waorani en las cuadrillas petroleras ha sido baja, ha constituido una fuente de ingresos monetarios que ha estado presente desde mediados de la década de los 70's. En las comunidades de estudio, la relación con esta actividad ha sido amplia.

Junto con el petróleo llegó una gran cantidad de personas de todo el país que para los waorani habían sido considerados como perjudiciales para su pueblo y supervivencia (Cabodevilla, 1999; Narváez, 2008). Dentro de estos grupos llaman la atención los kichwas amazónicos, quienes tenían una larga relación de tensión y violencia permanente con los waorani ya desde la época del caucho ${ }^{3}, \mathrm{y}$ desde el contacto cobraron importancia estratégica en la socialización del "mundo de afuera".

Otra actividad económica relevante, que se ha desarrollado en los últimos 30 años, es el turismo. Dentro del territorio waorani, esta actividad se ha desarrollado bajo el tipo de "ecoturismo" (Calderón, 1998) y ha respondido a la necesidad de relacionarse con el mundo externo desde la salida del ILV. Los precursores de la actividad fueron Sam Padilla (Caento), hijo de Dayuma, la cofundadora de Tiweno y Zoila (Wiñame) quien fue compañera de Dayuma y en 1971 salió de Tiweno para fundar Dayuno. Esta última comunidad se convirtió en el primer asentamiento waorani en atraer gran cantidad de turistas, que venían como mochileros principalmente de Estados Unidos y Europa (Stoll, 1985: 433). En la actualidad Cononaco Bameno y Keweiriono son las comunidades que han desarrollado la actividad más ampliamente como una posibilidad de ingresos económicos alternativos a las actividades extractivas.

\section{Las comunidades del estudio}

Keweiriono y Gareno son comunidades que se formaron entre principios y mediados de la década de 1990. Varios miembros de las dos comunidades tienen lazos de parentesco consanguíneo o ritual entre sí, y fueron parte del mismo grupo waomoni. Algunos habitantes de Gareno incluso vivieron en Keweiriono, pero salieron ya sea por conflictos internos, cuidado del límite occidental del territorio waorani, búsqueda de fuentes de trabajo asalariado (principalmente en el sector petrolero) o para acceder a centros educativos y comerciales.

Las dos comunidades tienen actividades económicas de autosubsistencia y asalariadas, sin embargo, la forma en que llevan las mismas son diferentes y en algunos casos diametralmente opuestas. En Keweiriono, la variedad de especies para la cacería es más abundante, debido en gran parte al cuidado que da la comunidad a su entorno y a la presencia de una zona enmarcada en un plan de amortiguamiento y protección del medio ambiente. Los miembros de la comunidad también manifiestan que la ausencia de la actividad petrolera ha contribuido a que los animales no hayan huido de la zona. En Gareno, comunidad donde se encuentran dos pozos petroleros (Wapuni y Nemonka), los animales que tradicionalmente se cazaban han disminuido considerablemente y los pobladores han incorporado otras variedades de animales que en el pasado no se consumían.

A pesar de que la pesca es una actividad relativamente nueva, se practica en las dos comunidades, sin embargo, en Keweiriono se registró un mayor número de especies y mayor

3 La cual estuvo marcada por varias muertes de ambos bandos. Los caucheros en su afán de abrirse campo entre la selva, buscaban más mano obra barata y si no podían dominar a los indígenas de estas zonas, ordenaban su muerte para continuar sus prospecciones. 
cantidad que en Gareno, debido principalmente que en la última comunidad los ríos aledaños han sido contaminadas por aguas residuales de la operación petrolera. La horticultura, por su parte, es mucho más extendida en Gareno y una parte de la producción se destina para la venta en los mercados aledaños (Feria de "San Pedro") o en la comunidad misma. A parte del cultivo de yuca y plátano, que son considerados como tradicionales, en esta localidad se ha incluido el cacao y el arroz. En Keweiriono, esta actividad se destina en su mayoría para el autoconsumo y una baja cantidad se vende en el "Waorani Ecolodge", que se encuentra a pocos minutos de la comunidad.

\begin{tabular}{|l|l|c|c|}
\hline \multicolumn{1}{|l|}{ Tabla 1: Datos Sociodemográficos de las Comunidades del Estudio } \\
\hline & & Keweiriono & Gareno \\
\hline Provincia & & Orellana & Napo \\
\hline Extensión & & $6 \mathrm{~km} 2$ aprox. & 45 \\
\hline \multirow{2}{*}{$\begin{array}{l}\text { Población Total } \\
\text { para 2009) }\end{array}$} & Hombres & 41 & 48 \\
\cline { 2 - 4 } & Mujeres & 26 & 93 \\
\cline { 2 - 4 } & Total & 67 & El Tena \\
\hline Ciudad más cercana & & El Coca & Terrestre \\
\hline & Forma de acceso & & \\
\hline
\end{tabular}

En relación a las actividades asalariadas, los hombres de las dos comunidades han trabajado para compañías petroleras. De todos los trabajos asalariados que han realizado los hombres entrevistados, el 57\% en Keweiriono y el 80\% en Gareno, están relacionados con el petróleo.

Sin embargo, para el tiempo del trabajo de campo, la mayoría de las personas de Keweiriono que participaban en actividades asalariadas lo hacían en el campo del turismo. En cambio, en Gareno, la actividad petrolera era la opción de trabajo más frecuente.

En relación a la infraestructura educativa, en Keweiriono existía una escuela y un colegio, que funcionaba con dos docentes. Los materiales educativos eran proporcionados por la empresa encargada de las relaciones comunitarias de la petrolera Repsol. En Gareno, existía una escuela que fue donada por la compañía petrolera Perenco, pero que funcionaba irregularmente. A 20 minutos en automóvil, se ubicaba el colegio "Sumak Sacha" que funcionaba a manera de internado.

Con respecto al tema de salud, en Keweiriono existía una promotora de salud y dos personas que se estaban capacitando para el mismo fin. En Gareno, había otra promotora de salud y existía un puesto de salud en la base de la compañía petrolera. Los casos graves de referían al Hospital del Tena. Los medicamentos, en las dos comunidades, eran entregados por la empresa encargada de las relaciones comunitarias de la petrolera Repsol.

\section{Breve introducción al concepto de identidad}

En el presente texto, se entiende a las construcciones identitarias de los hombres waorani como fenómenos dinámicos, que son resultado de procesos sociales y reflejan el devenir de contraste, negociación, y readecuación de un pueblo en relación a sí mismo y a la alteridad (Pujadas, 1993; Rival, 2000; Cardoso de Oliveira, 2007).

En el caso waorani, el contacto directo con agentes externos desde finales de los años 50, incorpora nuevas estrategias de identificación o selección frente a la alteridad que se visibilizan al analizar las relaciones entre individuos y grupos de diversas procedencias "nacionales", "raciales" o "culturales" y que Cardoso de Oliveira (2007) denomina como "contacto interétnico". A partir de las fluctuaciones que se dan en estas relaciones, se va formando una "cultura de contacto" que 
implica el conjunto de representaciones que un grupo étnico construye a partir de la situación de contacto en que está inmerso, y en términos del cual se clasifica a sí mismo y clasifica al otro.

En el nivel macro, durante los últimos 50 años, la cultura de contacto de los waorani se ha venido articulando a partir de dos instancias: la nación y la globalización. En este sentido, la nación como emisora de un conjunto de prácticas, valores, responsabilidades, derechos y normas de comportamiento (Hobsbawn, 2002) ha entrado en negociación con los grupos Waorani.

La globalización, por su parte, se entiende como un factor que trasciende el plano y que implica que la identidad se reelabore en un sentido intercultural denominado desterritorialización (García Canclini en Mantecón, 1993: 81). Esto ocurre debido a que las condiciones de producción y consumo en la actualidad no se dan en una sola sociedad, sino en circuitos globales los cuales llegan hasta los hombres waorani a través del trabajo asalariado, los medios de comunicación y la movilidad de los grupos (turismo, migraciones, escuela, entre otros factores) (García Canclini, 1990).

Al hablar de nación y espacios globalizados, no se puede afirmar simplemente de los grupos étnicos como receptores de mensajes externos, sino también como emisores. Mantecón (1993) señala que la globalización promueve no solamente los procesos de homogeneización, sino que también se han reforzado y resignificado las identidades locales. La etnicidad, vista como un proceso continuo de conocimiento, evaluación e interpretación compartida de los recursos simbólicos en contextos específicos y cambiante, se ha conformado en un instrumento del grupo waorani para lograr propósitos económicos y políticos específicos.

\section{Formas de producción e identidad}

El grupo waorani en la actualidad practica dos formas predominantes de producción: de autosubsistencia y asalariada. La primera, es considerada como "tradicional" del grupo, mientras que la segunda se popularizó después de la salida de las misioneras evangélicas del protectorado de Tiweno, a finales de los años 70's.

Hasta el momento del contacto, las actividades de autosubsistencia, consistentes en cacería, recolección y horticultura de baja escala, se desarrollaban en un territorio específico sobre el cual cada grupo waomoni tenía derechos de uso exclusivos, validados a partir del constante transitar por el mismo (Rival, 1996). Los hombres, en sus partidas de cacería, reconocían permanentemente el terreno, y de haber intrusos, tomaban acciones hostiles. De ahí que se consideraban como guardianes y guerreros.

La cacería tiene especial importancia en la comprensión de la construcción de las identidades masculinas waorani. En primer lugar, aunque no es exclusiva de los hombres, es considerada una actividad predominantemente masculina. El aprendizaje de las destrezas de la cacería implica un proceso de socialización profunda dentro de las comunidades.

Yost (1981), menciona que una buena cantidad de energía era dada al aprendizaje del cazador en la cultura Waorani. Señala que los adultos tomaban muy en serio su rol de ayudar a los niños a desarrollar sus brazos, pulmones, mejillas, mirada, oído y resistencia. Para el momento de la pubertad, los jóvenes debían tener un extensivo conocimiento en las llamadas de los animales, hábitos de construcción de nidos, comportamientos en torno a la respiración de los animales, ciclos de alimentación y hábitats de un gran número de especies. Rival (2000: 327) señala que en este aprendizaje los adultos no dan órdenes a los niños, tampoco ejercen coerción o ejercitan algún tipo de presión física o moral, sino que sugieren o piden, sin molestarse si la respuesta es no.

Otro punto de importancia de la cacería es su característica aglutinadora. A más de generar espacios de socialización, donde se reúnen muchas personas a escuchar y aprender de la experiencia de los demás, la gran cantidad de carne de monte es, con frecuencia, motivo para organizar y preparar fiestas. Es más, fiestas importantes como los matrimonios o encuentros entre 
miembros emparentados, no se pueden dar sin la presencia de carne. En este contexto, la cacería como evento productivo cobra relevancia en función de los momentos sociales que acompaña.

Aunque ésta y otras actividades consideradas como "tradicionales" siguen vigentes hasta la actualidad con mayor o menor intensidad, a raíz del contacto las actividades productivas fueron cambiando.

La presión demográfica del protectorado de Tiweno sobre las fuentes alimenticias tradicionales, como la carne de monte, y la influencia kichwa llevaron a que dichos grupos amplíen su universo de consumo de animales, hasta ese momento considerados tabú, e influyeron de igual forma a valorar la agricultura. Todos estos cambios inciden en los valores que construían a los hombres waorani como tales.

Las reubicaciones que fueron promovidas por las misioneras evangélicas del ILV y formaron la población de Tiweno influyeron en que el valor del cuidado del territorio vaya cambiando. Principios como el mantenerse lejos de los ríos a manera de protección fueron desapareciendo con la práctica de la pesca y el nuevo patrón de asentamiento en las riberas. De igual forma, las estrategias de ataque y huida, que habían sido cruciales para el abastecimiento de herramientas y otros insumos "kowore" hasta el contacto (Cabodevilla, 1999), fueron decreciendo conforme este último avanzaba.

Las formas productivas han cambiado también y se ha introducido la forma de trabajo asalariado. En muchos casos, la imagen del guerrero quedó como una valoración de los hombres de tiempos antiguos o es utilizada como una forma de reafirmación cultural, pero casi no se la práctica.

La participación en el trabajo asalariado es resultado de una práctica sistemática de introducción de nuevas necesidades en las comunidades, proceso que se dio de una forma extraordinariamente rápida y violenta.

El tipo de participación en esta clase de trabajo ha traído consecuencias importantes en la construcción de identidad de los hombres. En Gareno, el tipo de relación laboral con las petroleras ha estado marcado por formas de trabajo temporales y poco especializadas. El poco involucramiento de la compañía con la comunidad fuera de la esfera económica ha generado que los agentes petroleros sean vistos como extraños que buscan su mayor rentabilidad a costa de la comunidad y el territorio. Estas prácticas se pueden enmarcar en situaciones de conflicto interétnico, es decir aunque los dos grupos interactúan en un territorio, pugnan permanentemente por los recursos y beneficios, pero son interdependientes entre sí. A esto Cardoso de Oliveira (2007) ha llamado fricción interétnica. Esto se hace más crítico debido a que las compañías han utilizado mucho la práctica de repartir beneficios entre pocos.

Por otra parte, muchos de los hombres de la comunidad trabajan como jornaleros para la empresa petrolera o para otras que se ubican en zonas más alejadas de la comunidad. Lo que lleva a los hombres de esta comunidad a trabajar en los espacios mencionados es la creciente demanda de artículos de fuera y la mayor relevancia de la educación formal, como mecanismo para lograr un mejor posicionamiento en relación al "mundo de afuera".

Frente a la cercanía de las petroleras, los hombres en Gareno han desplegado dos estrategias que se pueden observar en todo el grupo waorani: algunos buscan lograr un espacio preferencial en la negociación con las mismas, mientras que otros se anclan en sus costumbres y forma de vida ancestral para reclamar tipos de negociación más equitativos. Estas dos formas de pensar al otro generan también conflictos intraétnicos regidos por conjuntos de valores muchas veces opuestos. Mientras que algunos habitantes reclaman el derecho sobre su territorio y sobre el usufructo que se realiza sobre él, otros proponen participación en la toma de decisiones y acceso más generalizado a los beneficios.

Por otra parte, la actividad petrolera ha abierto un espacio muy grande para que los waorani se pongan en contacto con personas de todo el país, donde sí se han establecido relaciones de parentesco ritual que trascienden el momento de la actividad productiva. La empresa petrolera tendría dos rostros: uno difuso e institucional, donde no hay formas claras de establecer relaciones 
sociales más cercanas, y que tendría que ver con la institucionalidad de la empresa; el otro conformado por los obreros y en algunos casos relacionadores comunitarios, con quienes se han establecido lazos sociales más cercanos.

En Keweiriono, el petróleo también ha representado un espacio importante en la consecución de trabajos asalariados. Sin embargo, en los últimos 10 años, no ha sido ni el principal, ni el más cercano debido a que la actividad turística se ha posicionado como un espacio prioritario.

La imagen del otro en esta forma económica es diferente, ya que, por lo general, las personas que visitan la comunidad están interesadas en conocer otras formas de vida y pagan para verlas. Es así que la actividad turística, en Keweiriono y otras comunidades que han optado por esta práctica, ha sostenido mucho sus operaciones, a partir de la promoción de ciertas características culturales consideradas como atractivas para los visitantes (Calderón, 1998).

Si bien la manera de presentarse a los turistas muchas veces se encuentra rediseñada por la industria del turismo (Ibíd.), genera también que el extranjero sea visto en diferentes términos.

Por una parte, se hace una distinción más clara entre los tipos de extranjeros que visitan la comunidad. Se encuentran los turistas que llegan con el objetivo de conocer otra forma de vida, a quienes los waorani organizan espectáculos que contienen elementos de su tradición, les venden artesanía, en algunos casos trocan prendas de vestir u otros objetos, y les sirven en los espacios destinados a la actividad turística. Otros agentes son los trabajadores de la empresa turística. La relación con estos personajes es más continua y algunos habitantes de la comunidad tienen lazos de parentesco ritual con ellos, como el padrinazgo y compadrazgo. Sin embargo, esta última situación genera eventualmente conflictos internos por el acceso desigual a los beneficios de la actividad.

En este sentido, los hombres no son solamente contingente de mano de obra, sino que forman parte integrante de la práctica económica. Este tipo de actividad ha generado una mayor cercanía con los otros e implica reglas de juego más flexibles.

\section{Circulación, reciprocidad e identidades masculinas}

Las formas de circulación entre los waorani, dentro y fuera de su conjunto cultural, evidencian también las fronteras de la identidad. Por ejemplo, la circulación de la producción interna tradicionalmente se ha enmarcado en un contexto de reciprocidad generalizada, es decir, como una forma de intercambio donde lo que prima es la relación social sobre la situación "económica" que se experimenta (Sahlins, 1977). Hasta el momento del contacto, la reciprocidad generalizada era la única forma de intercambio y marcaba claramente quienes eran los propios y quienes los ajenos, ya que estaba negado el intercambio con grupos waorani lejanamente emparentados (warani) y con no-waorani. Para obtener bienes manufacturados externos, se valían de estrategias de incursión hostiles (Cabodevilla, 1999).

Desde el contacto, esta forma de reciprocidad ha tenido que cambiar debido a las nuevas relaciones que se han ido articulando. Así a la reciprocidad generalizada, se han sumado la equilibrada y la negativa, que reflejan distintos niveles de cercanía entre actores.

En términos de Sahlins (1977: 212), reciprocidad equilibrada se entiende como las

"transacciones que estipulan una retribución de valor o utilidad conmensurados dentro de un período finito y no muy largo". Por su parte, la reciprocidad negativa es la práctica mediante la cual los participantes se enfrentan como intereses opuestos, tratando cada uno de obtener el máximo de utilidad a expensas del otro (Ibíd.: 213-214).

En el proceso de negociación con agentes externos y el consiguiente aparecimiento de nuevas formas de reciprocidad dentro el pueblo waorani, los hombres han jugado un papel importante, ya que, en el plano laboral y de organización, han sido quienes más han participado. Algunos hombres que en Tiweno accedieron a la educación formal y que estuvieron cerca de las 
misioneras, se convirtieron posteriormente en intermediarios y negociadores con las compañías petroleras y con otros actores que llegarían posteriormente.

La ONHAE y posterior NAWE, por primera vez en la historia del grupo, funcionó como entidad de representación de todo el conglomerado waorani. Esta nueva forma de organización, a diferencia del sistema profundamente horizontal que mediaba las relaciones entre los diferentes miembros de las familias y grupos afines waorani, propiciaba una directiva que hablaba en representación del conglomerado.

La circulación de beneficios procedentes del petróleo es un tema conflictivo dentro de las comunidades. Por ejemplo, aunque el discurso petrolero, desde la aparición de la empresa MAXUS (1993), fue el de beneficiar a las comunidades waorani con la explotación petrolera y mejorar las condiciones de salud, educación, entre otras en el área; en la práctica utilizó una estrategia consistente en beneficiar a pocas personas que ayudaran a la entrada y validación de las compañías en las comunidades (Narváez, 2008; Rivas, 2001).

Esta práctica se puede enmarcar en una situación de reciprocidad negativa, donde la compañía, a través de sus departamentos de relaciones comunitarias, pretendía lograr la posibilidad de explotación con un bajísimo costo de inversión social.

El conflicto que esta situación ha generado es que, tanto a nivel interno como externo, las organizaciones de representación no han distribuido al conjunto de las comunidades los beneficios de la actividad. Sin embargo, se propone que históricamente no existió nada parecido a la idea occidental de representación. Se sugiere que los valores que occidente atribuye a un buen ejercicio de liderazgo, como puede ser la distribución equitativa de bienes conseguidos en las negociaciones, son valores ajenos. En varias ocasiones, tiene más peso el compromiso con los grupos más cercanos (nanicabos o grupos waomoni), que la responsabilidad adquirida frente a todo el grupo waorani, que muchas veces pertenece a grupos lejanos. Se presenta entonces una situación de fricción intraétnica que involucra dos éticas que se encuentran en pugna.

En el caso del turismo, la situación con respecto a la circulación de las ganancias tiene una dinámica diferente. En Keweiriono, por ejemplo, aunque la negociación se mantiene a través de un representante de la comunidad, la actividad turística se ha pensado más como comunal, y la mayoría de personas participan de alguna forma.

Por otra parte, en todas las comunidades waorani, la circulación de bienes por medios monetarios, es otro factor que se ha ido popularizando en los últimos 50 años, pero que tiene sus particularidades de acuerdo a las comunidades de estudio. En Gareno, por ejemplo, las transacciones monetarias son muy frecuentes debido a la presencia más cercana de centros de comercio. Esto afecta a las dinámicas de reciprocidad generalizada, ya que algunos productos que se destinaban al intercambio interno y al fortalecimiento de relaciones, en la actualidad son vendidos. En Keweiriono, si bien se saca carne para la venta, la mayoría de ella se consume dentro de la comunidad, alimentando en diversos sentidos la reproducción del grupo.

De esta forma, desde la mirada de la autoidentificación, mientras que en Keweiriono los hombres se siguen viendo como cazadores, en Gareno han pasado mirarse como agricultores o empleados, situación que trae una serie de problemas sociales y psicológicos al interior de la comunidad (alcoholismo, violencia doméstica). Otro cambio ha sido la resignificación de algunos artículos externos en la práctica de reciprocidad generalizada, pero también en la reciprocidad equilibrada.

\section{Consumo e identidades masculinas}

El tema de consumo e identidad se aborda desde el tema de alimentación, pero también de manifestaciones culturales. En relación al primer componente, se ha manifestado que la carne de monte, por ejemplo, ha acompañado momentos sociales muy importantes como fiestas y matrimonios. La carne de monte ha nutrido espacios de reproducción social del grupo. 
A pesar de que algunos alimentos que se consiguen en el mercado pueden incorporarse a las redes de reciprocidad, el lugar de la carne en las celebraciones o eventos sociales relevantes, sigue siendo fundamental. El caso de Gareno, comunidad donde los animales han disminuido considerablemente, refleja que la ausencia de carne afecta la consecución de eventos de reunión comunal que alimente el sentimiento de comunidad.

“Antes se compartía más, porque en ese tiempo, hace 30 años había más carne y pesca...Uno llegaba a las casas y cualquier carne nos brindaban. Ahora es menos porque hay menos carne, los animales se van y ya no se puede compartir" (David Andi, Gareno, 2009).

En Keweiriono la situación es diferente. A pesar de que existe un consumo de alimentos de afuera, el abandono de prácticas tradicionales como la cacería, es mal visto dentro de la comunidad. Se mantiene entonces como un elemento de importancia en la constitución de un hombre como tal.

"Él trabaja bastante en las cabañas. Entonces la gente anda diciendo que es vago por no cazar, que ya no parece waorani, solo comida de blanco come. Por eso es que ha salido ahora, porque él si sabe cazar" (Obe, Keweiriono, 2009)

Finalmente las identidades y el consumo no solo de elementos productivos o vituallas, sino también de manifestaciones culturales. El tipo de consumo depende en gran medida de la relación que los habitantes de cada una de las comunidades, han establecido con factores externos. En Keweiriono, por ejemplo, el contacto con los turistas ha provocado que la gente waorani acceda a objetos como gafas de sol, linternas, mochilas o ropa, pero también que conozcan otras formas de vida.

Por otra parte, algunos de los mensajes que se consumen a través de la televisión y la radio, principalmente, influyen en la vestimenta y preferencias de consumo de los jóvenes.

La escuela por su parte, es un factor fundamental para generar nuevos consumos entre los waorani. Paralelamente a las necesidades que esta actividad demanda (útiles escolares, ropa), la educación oficial va generando nuevas necesidades materiales sociales e intelectuales.

\section{Conclusiones}

El proceso de reflexión sobre las identidades de los hombres waorani en las comunidades de Keweiriono y Gareno, puede ser entendido a partir de un estudio histórico, procesual y relacional de la situación waorani- economía de mercado. Se ha identificado que estos elementos están directamente relacionados en los espacios laborales, de relaciones humanas, resignificación del territorio, consumo y dotación de bienes. Indirectamente, la influencia del mercado en las identidades waorani, se manifiesta en los nuevos sistemas de socialización y educación, relaciones intrafamiliares, roles y prioridades de los hombres, estados de ánimo, entre otros puntos.

La identidad actual de los hombres waorani, en este sentido, es producto de un proceso histórico en el cual ha influido la guerra, el contacto con los misioneros, las petroleras, el turismo y las diferentes influencias externas que han recibido desde fines de la década de 1950. Los factores mencionados han traído consigo formas particulares de relaciones laborales, uso de dinero, escolarización, bienes, alimentos, todos los cuales han sido negociados de maneras específicas por las comunidades del estudio. La diferencia radicaría en que mientras Keweiriono ha optado por elecciones más cercanas a elementos de su tradición, Gareno, ha apostado por las formas de vida externa. Estas diferentes opciones, repercuten directamente en las prioridades de los hombres.

El trabajo de campo en las dos comunidades constató que además de la escolarización, otros espacios donde se presenta una reconfiguración de la identidad son los laborales, de relación con 
el medio ambiente, sociales y de consumo. En todos ellos, confluyen las diferentes dimensiones de la realidad social del pueblo waorani y se construyen las mencionadas culturas de contacto.

En este sentido, también se identificó, que los niveles más cercanos de reciprocidad, están directamente relacionados con relaciones más afianzadas y que en ese sentido situaciones de reciprocidad generalizada correspondían a la familia, amigos o afines. Por otro lado, situaciones de reciprocidad negativa se dirigían a relaciones de conflicto.

Para concluir, es necesario recordar que las prácticas económicas, en general, y las relacionadas con el sistema de mercado, en particular, influyen y se negocian permanentemente en los hombres waorani, a partir de sus valores e ideologías, que son elementos de la identidad. Por lo tanto, sirven de espejo para analizar este fenómeno en los tiempos que corren.

\section{Bibliografía}

Cabodevilla, M. 1999. Los Huaorani en la Historia de los Pueblos del Oriente. Quito : Cicame.

Calderón, M. 1998. Oil Transnationals and the Huaorani community of the Amazon basin: Redefining Development in the Ecuadorian Rainforest. Baltimore County: Universidad de Maryland.

Cardoso de Oliveira, R. 2007. "Etnicidad y Estructura Social”, Clásicos y Contemporáneos en Antropología. México.

Cardoso de Oliveira, R. 1998. Etnicidad, Eticidad y Globalización”. En Bartolomé A., Barabas, M, Barabas, A. (Ed). Autonomías étnicas y Estados nacionales.

Clastres, P. 2001. Investigaciones en Antropología Política. España: Gedisa. España.

García Canclini, N. 1990. Culturas Híbridas. Estrategias para entrar y salir de la modernidad. México: Alianza Editorial.

Labaka, A. 2003. Crónica Huaorani. Quito: Cicame.

$\mathrm{Lu}$, F. 2007. Integration into the Market among Indigenous Peoples. The Werenr-Gren Foundation for Anthropological Research.

Mantecón, Ana Rosas. 1993. Globalización cultural y antropología. En Alteridades, 79-91.

Narváez, I. 2008. Huaorani: mundos paralelos, mundos superpuestos y submundos.

Pujadas, Juan José. 1993. Etnicidad, Identidad Cultural de los Pueblos. Madrid: Eudema.

Rival, L. 2000. La escolarización formal y la producción de ciudadanos modernos en la Amazonía ecuatoriana. En Guerrero, A. (comp.). Etnicidades. Flacso-Ecuador, Quito: ILDIS. Serie Antología.

Rival, L. 1996. Hijos del Sol, Padres del Jaguar. Quito: Ediciones Abya-Yala.

Rivas, A; Lara, R. 2001. "Conservación y Petróleo en la Amazonía Ecuatoriana”, Quito: Ecociencia/Abya-Yala,.

Sahlins, M. 1977. Economía de la Edad de Piedra. Madrid:, Akal Editores.

Sánchez- Parga, J. 1992. Identidades y sociedad. Quito: Centro de publicaciones CELA.

Smith, R. 1996. Drama Bajo el Manto Amazónico. Quito: Abya Yala.

Stoll, D. 1985. Pescadores de Hombres o Fundadores de Imperio. El Instituto Lingüistico de Verano. Quito: Abya Yala.

Yost, J. 1979. El Desarrollo Comunitario y La Supervivencia Étnica. Cuadernos Etnolingüísticos N 6, Quito.

Yost, J. 1981. Veinte Años de Contacto: Los Mecanismos de Contacto en la Cultura Huao (Auca). Cuadernos Etnolonguísticos No.9, Quito. 